\title{
Edge diffraction of a dipping reflector: an analysis with a GPR dataset
}

\author{
Susanne Maciel*, ${ }^{1}$ Amanda Rocha, ${ }^{2}$ and George Sand França ${ }^{2}$ \\ 1) FUP/UnB \& INCT-GP \\ 2) $I G / U n B$
}

\section{ABSTRACT}

Diffracting edges may indicate geologic faults and possible associated traps, thus being of great interest for seismologists. However, diffraction amplitudes present characteristic signatures in seismic records and radargrams, and are usually proportionally smaller in magnitude than refletcion events. We present a specific preprocessing method for diffraction enhancement that preserves diffraction patterns, based on filtering in a specific domain. The method uses the most important concepts of signal analysis for undergraduate students. We describe a simple physical experiment to analyze edge diffractions generated by a zinc sheet buried in a sandbox in a radargram acquisition. Results of this experiment are presented, and a didatic exposure is proposed for geophysics students from University of Brasilia.

\section{INTRODUCTION}

The theory of seismic wave propagation in acoustic media is used to transform seismograms into realistic Earth models. Traditional processing of seismic data generally uses information from the reflected wavefield to obtain images from interfaces at the subsurface. An important aspect when interpreting subsurface images is the identification of small-scale features, such as faults, channels, and fractures. Instead of promoting reflections, the seismic energy interacting with these structures results in diffractions, when their dimensions are smaller than the acoustic wavelength emitted during seismic acquisition. This information can be used for high-resolution imaging (Khaidukov et al. (2004)) or local velocity analysis (Reshef and Landa (2009)).

Diffracted seismic waves have different characteristics than reflected ones. Trorey (1970) showed that for a single truncated plane reflector, the phase of the diffractions suffers a reversal of $180^{\circ}$ on either size of the reflecting edge. Scattered waves are recorded as significantly lower energy than reflected waves. The amplitude of a diffraction from a single edge decays faster than it would be by simple inverse spreading, and for this reason, diffrac- tions usually are treated as noise in traditional seismic processing. In isotropic media, diffraction traveltimes can be approximated by a conventional double-squareroot (DSR) equation. The amplitudes extracted along the elementary diffractions, known as the diffraction operator, forms a curve that is used by Tabti et al. (2004) to determine the so-called Fresnel aperture, used to enhance Kirchhoff-type depth-migration. In common-offset sections, the diffraction operator of an image point is distinct between reflections, diffractions or void points.

\section{METHOD}

As described by Tabti et al. (2004), a diffraction operator $D(M, \xi)$ at a generic image point $M$ is defined as the vector of all seismic amplitudes to be stacked by a Kirchhoff migration for point $M$ and midpoint coordinate $\xi$. In other words, it is given by

$$
D(M, \xi)=\left.W(M, \xi) \partial_{t} U(\xi, t)\right|_{t=\tau_{D}(M, \xi)}
$$

as a function of position $\xi . W(M, \xi)$ is a weight function, $U(\xi, t)$ is the seismic data measured at position $\xi$, and $\tau_{D}(M, \xi)$ is the traveltime of elementary diffraction of $M$. In this study, we use a simplified version of the diffraction traveltime, where $\tau_{D}$ is calculated only for isotropic media, with small lateral variations on the velocity model, which brings a double-square root approximation

$\tau_{D}(M, \xi)=\sqrt{\left(\frac{t_{0}}{2}\right)^{2}+\left(\frac{\xi-h}{v_{r m s}}\right)^{2}}+\sqrt{\left(\frac{t_{0}}{2}\right)^{2}+\left(\frac{\xi+h}{v_{r m s}}\right)^{2}}$

where $t_{0}$ is the zero-offset two-way time for image point $M, h$ is the half-offset and $v_{r m s}$ is the RMS velocity.

Note that Kirchhoff migration consists in simply attributing to point $M$ the sum over $D(M, \xi)$ for all values of $\xi$ within the migration aperture.

The diffraction traveltime curve associated with an image point located on a reflection is tangent to the reflection traveltime at the specular reflection event. This point becomes a tangential region when the source has limited bandwidth, which is defined by Tabti et al. (2004) 


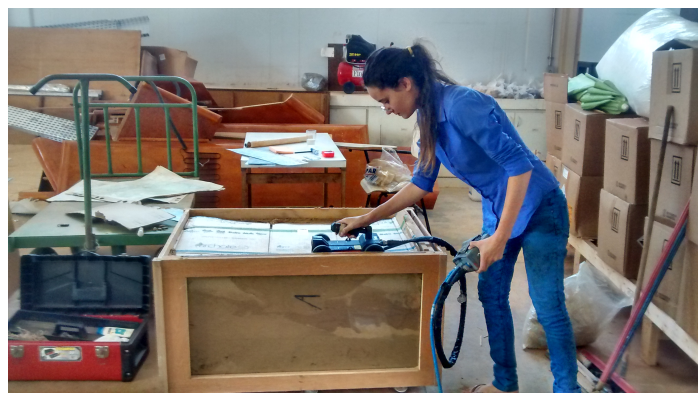

FIG. 1. GPR acquisition on a sand box.

as Fresnel aperture, and by Schleicher et al. (1997) as the minimum aperture for true-amplitude depth migration.

\section{A. GPR experiment with a sand box}

The problem of building a good algorithm for modeling diffractions is tricky (Moser 2012). Since diffractions are scattered in all directions, ray-based modeling does not address the problem of diffraction propagation. Kirchoff modeling is able to predict kinematic aspects of diffractions, but not the dynamic aspects. Keller (1962) described the geometric theory of seismic diffractions, but implementing a modeling algorithm with his ideas might be somewhat complicated. This way, we developed a method to make the acquisition on a very controlled environment. With a GPR antenna of $2600 \mathrm{MHz}$, and with a wooden box filled with sand, we are able to acquire radargrams on an extremely controlled environment. The box has dimensions $100 \mathrm{~cm} \times 60 \mathrm{~cm} \times 80 \mathrm{~cm}$ (Figure 1$)$.

In the present work, we present the experiment of analyzing edge diffractions generated by the corner of dipping reflectors (Figure 2).

\section{B. The Scattering Moveout corrected Common Offset gather}

A concept to be discussed is what we mean by scattered energy and diffracted energy. Scattering is a general physical process where energy, in our case, acoustic energy, is forced to deviate from a straight trajectory by one or more paths due to localized non-uniformities in the medium through which they pass. This also includes deviation of reflected waves from the angle predicted by Snell law. Diffractions are energy scattered in all directions and therefore kinematically visible regardless of the acquisition aperture.

Consider a diffraction event on a common offset gather at point $p$, located at $\left(x_{D}, z_{D}\right)$. For an isotropic media, we know that the traveltime of the event is the hyperbola given by equation (2), whose apex is located at $x_{D}$. The difference between the two-way time at a given midpoint $(\xi)$ and the two-way time at $x_{D}$ is called here scat-
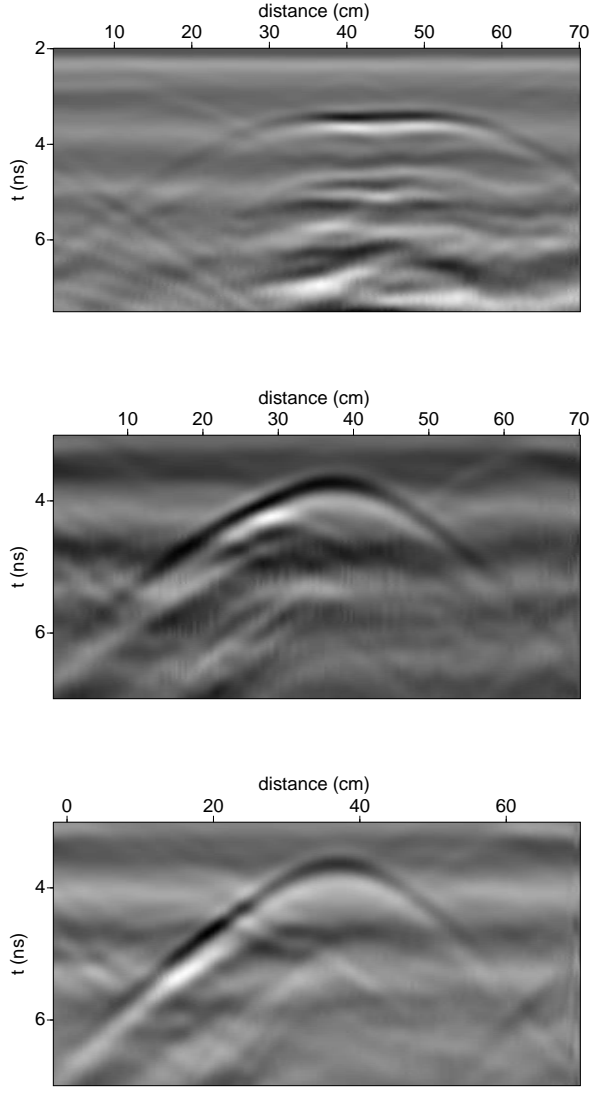

FIG. 2. GPR aquisition of plane zinc sheets reflecting with $\operatorname{dips} 0^{\circ}, 30^{\circ}$ e $45^{\circ}$.
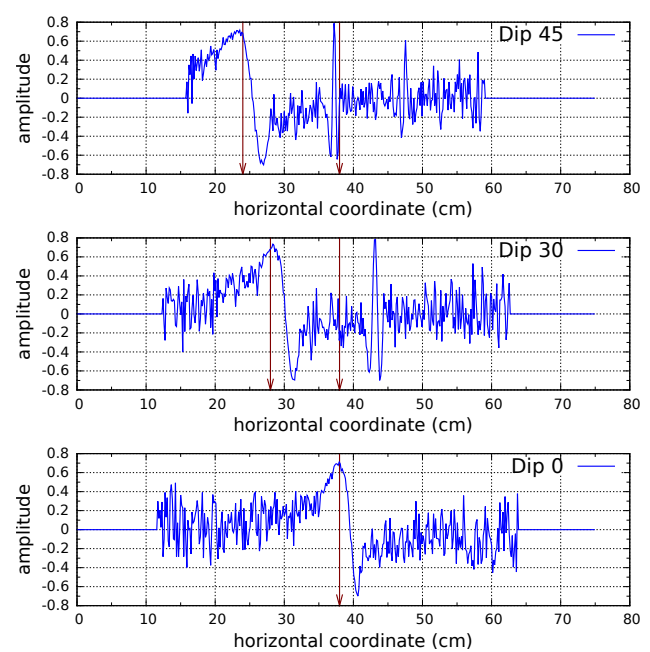

FIG. 3. Diffraction operators associated with edges from plane reflectors with dips $0^{\circ}, 30^{\circ}$ e $45^{\circ}$, indicated on Fig. 2. Arrows indicate the distance between specular event and diffractor horizontal coordinate. 


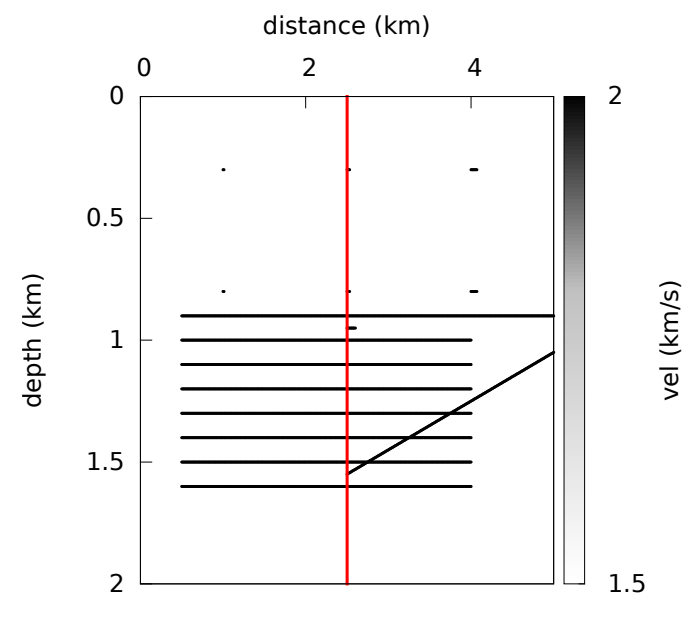

FIG. 4. A synthetic velocity model. Vertical profile indicated in red.

tering moveout (SMO). SMO depends on velocity above the scatterer, offset and the two-way traveltime associated with the midpoint upon the scatterer coordinate. Similar to a NMO correction procedure, once the velocity model is estimated, the traveltimes can be corrected to remove the effect of scattering moveout, as shown in Figure 5(a). If traces in the SMO-corrected gather are summed up, one obtains a Kirchhoff migrated trace at the particular CMP location.

SMO-corrected gathers (Figure 5(a)) are used by Tabti et al. (2004) to perform true-amplitude Kirchhoff migration. They are used to visualize Fresnel aperture (or projected Fresnel zone) since high amplitudes indicate the size of the aperture. For our case, SMO-corrected gathers are useful because they display horizontally all diffraction operators at a given vertical profile.

For instance, Figure 5(a) is constructed from diffraction operators of image points along the profile indicated in red. If we plot the amplitudes along a horizontal slice (constant-depth point) in Figure 5(a), we obtain the diffraction operator corresponding to that subsurface location (i.e. similar to the curves shown in 3).

Figure 2 shows three radargrams discontinuous dipping reflectors, all beginning at coordinate $38 \mathrm{~cm}$. Depending on the local dip of the corresponding reflector, the Fresnel aperture segments will be offset from the profile position either to the left or to the right. The amount of offset will, in general, depend on the dip, the depth of the reflector and the complexity of the overburden model.

\section{Filtering}

When a diffraction hyperbola crosses reflection events, or other scattering events, the corresponding diffraction operator has peaks of amplitudes which are not related to the image point, leading to a complicated pattern in the SMO corrected gather. These peaks may be removed by
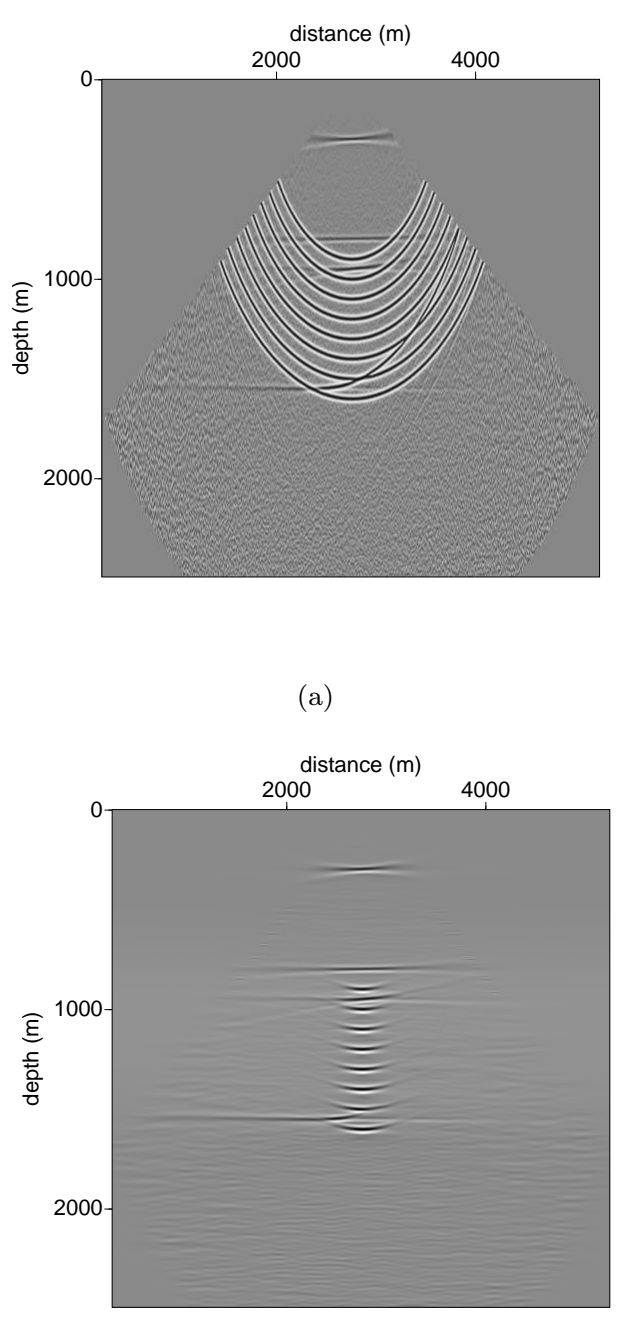

(b)

FIG. 5. SMO corrected CO gather before (top) and after (bottom) slope filtering.

applying some kind of filter on the Scattering Moveout corrected gather. Tabti et al. (2004) proposed the application of low pass filtering, which can remove undesirable peaks. On the other hand, it might not be suitable for our purposes since low pass filtering may also destruct the original pattern of a diffraction operator shape, leading to further misclassification of the image point.

As explained earlier, Fig. 5(a) shows us that the interesting information is displayed horizontaly in the SMO gather. A horizontal profile at a certain depth gives us the diffraction operator associated with that depth. Any coherence observed in directions distinct from horizontal is associated with crossing events along the elementary diffraction traveltime. This fact inspired us to apply not a low pass filtering on the SMO gather, but a slope filtering, removing any coherent energy that is not horizontal (see Figure 5). 


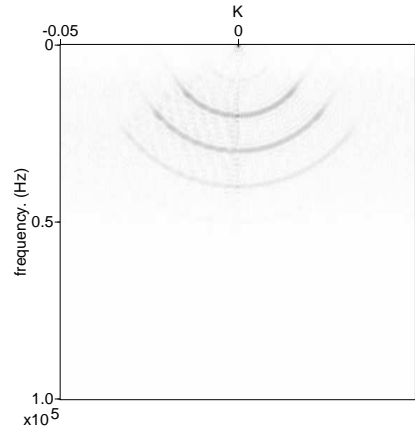

(a) F-K Fourier spectrum.

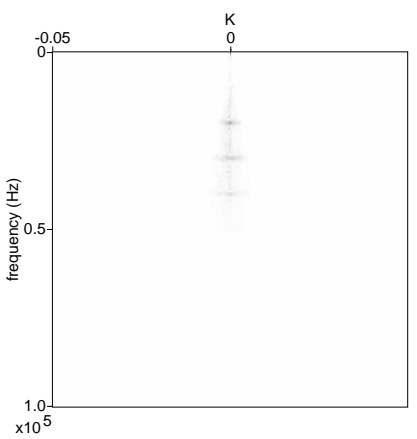

(b) Slope filtered F-K spectrum.

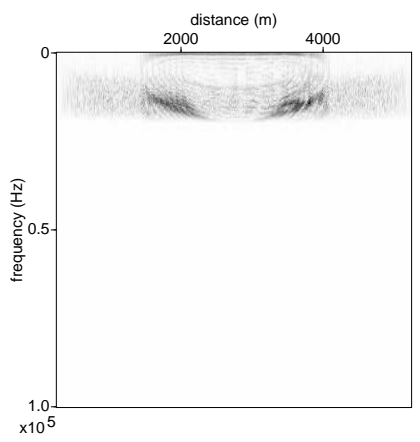

(c) Low-pass filtered F-X Fourier spectrum.

FIG. 6. F-K Fourier spectrum of Scattering Moveout corrected gather showed in Figure 5 (left), and application of slope filtering (center). The effect of low-pass filtering is shown in F-X Fourier spectrum (right).

Figure 6 shows how it works. We first apply a F-K Fourier transform on the SMO gather, and then we remove all frequencies within a reason $|K / F|$ bigger than 0.3 (nearly horizontal). Back to time domain, we obtain Figure 5(b).

\section{DIDACTIC TRANSPOSITION}

Coherence, slope filtering and low pass filtering are basic concepts that are part of the discipline Signal Analy- sis, from the Geophysics course at University of Brasilia. The physical experiment is simple and fast to be implemented with students, and it requires a GPR antenna, a control system and a sandbox. The pre-processing of the data is performed using the software Reflex by the tutors of the discipline, and once the data is available in ASCII, students are able to perform the filtering procedure described in Section III C using Python. The theory and the pseudo codes are worked in classroom, and the students are asked to develop their own filters based on the codes developed previously. Other objects can also be buried to obtain variated signal patterns. Another variation can be obtained by making the sand wet, in order to provide others dieletric constant for the propagation medium.

Concepts such as diffraction imaging, migration and acquisition geometries are not necessary to implement the filtering procedure, if these are concepts not yet developed within the current class.

\section{RESULTS AND CONCLUSIONS}

GPR datasets are extremely useful for diffraction studies, and the sandbox experiment is of great usefulness for didactic aspects on seismic processing lectures, imaging tehory and signal analysis. Save for the presence of a GPR antenna, the experiment is easy to be reproduced by any teaching lab. Results show that the slope filtering technique using SMO gathers are useful for diffraction imaging procedures, since it preserves diffraction signatures in a better way when compared to low-pass filtering. Students may beneficiate from physical experiments when attending Signal Analysis discipline, where they are able to identify a whole workflow for signal processing, using both novel and traditional applications of processing.

Keller, J. B. (1962). Geometrical theory of diffractions. Journal of the Optical Society of America, 52:116-130.

Khaidukov, V., Landa, E., and Moser, T. J. (2004). Diffraction imaging by focusing-defocusing: An outlook on seismic superresolution. Geophysics, 69:1478-1490.

Moser, T. J. (2012). Review of ray-born forward modeling for migration and diffraction analysis. Stud. Geophys. Geod., 56:411432 .

Reshef, M. and Landa, E. (2009). Post-stack velocity analisys in the dip angle domain using diffractions. Geophysical Prospecting, 57:811-821.

Schleicher, J., Hubral, P., Tygel, M., and Jaya, M. S. (1997). Minimum aperutres and fresnel zones in migration and demigration. Geophysics, 62:183-194.

Tabti, H., Gelius, L., and Hellmann, T. (2004). Fresnel aperture prestack depth migration. First Break, 22:39-46.

Trorey, A. W. (1970). A simple theory for seismic diffractions. Geophysics, 35:762-784. 